\title{
Projeto Integrado de Controle de Pelotões baseado em Consenso e um Protocolo de Disseminação de Dados Confiável
}

\author{
Renê Oliveira ${ }^{1}$, Carlos Montez ${ }^{1}$, Michelle S. Wangham ${ }^{2}$ \\ ${ }^{1}$ Depto de Automação e Sistemas - Universidade Federal de Santa Catarina (UFSC) \\ ${ }^{2}$ Mestrado em Computação Aplicada - Universidade do Vale do Itajaí (UNIVALI) \\ rene.oliveira@posgrad.ufsc.br, carlos.montez@ufsc.br, wangham@univali.br
}

\begin{abstract}
Automated and coordinated platoon driving involves theories of control, vehicle dynamics and vehicular communication. Due to a constant interaction between control logic and communication topology, a co-design approach to the controller and the communication protocol becomes necessary. This article introduces AddP-CACC - a consensus-based vehicle platoon solution, in which topology is a design parameter that can be exploited to reconfigure the controller, depending on the characteristics of the scenario. AddP-CACC was compared to other techniques, and the results show its robustness and ability to maintain a stable vehicle platoon in different communication topologies.
\end{abstract}

Resumo. A condução automatizada e coordenada de pelotões envolve teorias de controle, dinâmica dos veículos e comunicação veicular. Devido a uma constante interação entre a lógica de controle e a topologia de comunicação, uma abordagem de co-projeto para o controlador e o protocolo de comunicação torna-se necessária. Este artigo apresenta a AddP-CACC - uma solução para pelotões de veículos baseada no consenso, na qual a topologia é um parâmetro do projeto que pode ser explorado para reconfigurar o controlador, dependendo das características do cenário. A solução foi comparada a outras técnicas da literatura e os resultados obtidos comprovam sua robustez e capacidade em manter um pelotão veicular estável em diferentes topologias de comunicação.

\section{Introdução}

As redes veiculares cumprem papel fundamental nos Intelligent Transportation Systems (ITS), suscitando pesquisas em aplicações associadas ao conforto, eficiência e segurança no tráfego [Lyamin et al. 2018]. A condução automatizada é uma área ativa de investigação que visa aumentar a segurança, reduzir congestionamentos e emissões de gases dos veículos. Nessa área de investigação, a implementação de pelotões inteligentes (vehicle platooning) que agrupam veículos visa ajustar automaticamente a velocidade de cada veículo de modo a manter uma distância segura entre eles.

Problemas semelhantes aos de controles de pelotões são encontrados em sistemas de controle via redes (NCS - Networked Control Systems) [Kheirandish et al. 2017]. Em ambos, o co-projeto do algoritmo de controle com o da rede de comunicação se mostra adequado e visa controlar o sistema e assegurar a estabilidade do sistema. No caso dos pelotões, essa estabilidade é caracterizada através do conceito denominado estabilidade em cadeia (string stability) [Schwartz et al. 2012], a qual considera a propagação de perturbações gerada por um veículo na direção dos veículos seguidores. Em um pelotão estável, um distúrbio não deve ser amplificado para os veículos seguidores. 
Devido aos requisitos de tempo e confiabilidade das aplicações de controle de pelotão e às características das comunicações veiculares (diferentes densidades de rede, mudanças rápidas de topologias e desconexões frequentes), um protocolo de disseminação de dados confiável é crucial. Neste artigo é proposto o AddP-CACC uma solução de controle para pelotões veiculares baseada em um algoritmo de consenso projetado especificamente para lidar com atrasos heterogêneos e variáveis no tempo da comunicação. O protocolo de disseminação confiável de dados AddP, proposto em [Oliveira et al. 2017], foi adaptado e integrado a um algoritmo de controle de pelotão.

A contribuição mais significativa deste artigo é a estratégia de controle cooperativo descentralizada baseada em consenso que, comparada aos trabalhos relacionados, é reconfigurável em tempo de execução e se adapta às condições da rede na presença de atrasos de comunicação. A abordagem de controle compensa automaticamente informações desatualizadas causadas por perdas e atrasos na rede. A avaliação do desempenho e da robustez da solução proposta, frente a perturbações na dinâmica do veículo líder e falhas de comunicação, foi conduzida neste trabalho por meio de simulações.

O restante deste artigo está organizado da seguinte forma. A Seção 2 contextualiza os tipos de automação veiculares e topologias de redes veiculares. A Seção 3 analisa os principais trabalhos relacionados. A Seção 4 apresenta a solução proposta e a Seção 5 descreve os resultados das simulações e os analisa. Finalmente, a Seção 6 conclui o trabalho com uma discussão sobre as contribuições e os trabalhos futuros.

\section{Contextualização}

Uma alternativa para aumentar o fluxo de veículos e evitar o congestionamento do tráfego é reduzir o espaçamento dos veículos através de um sistema automatizado [Elefteriadou 2013]. Para facilitar o desenvolvimento de sistemas de automação de veículos, a National Highway Traffic Safety Administration (NHTSA) definiu níveis distintos de automação [Hicks 2018]. O sistema de suporte de direção mais simples, com um certo nível de automação, é um controle longitudinal que mantém automaticamente um Cruise Control (CC) constante [Rajamani 2011]. O aprimoramento da tecnologia de controle de veículos deu origem ao sistema Adaptive Cruise Control (ACC). A distância entre veículos e a velocidade em relação ao veículo anterior são medidas usando sensores.

Sistemas ACC geralmente se concentram no conforto ao dirigir, e buscam manter uma distância de segurança comparável a de condutores humanos [Rajamani 2011]. A extensão do ACC, ao se acrescentar conectividade entre veículos e Road Side Units (RSUs), é denominada CACC (Cooperative and Adaptive Cruise Control). Veículos equipados com CACC não apenas medem a distância e a velocidade em relação ao veículo anterior, mas também podem se comunicar com outros veículos equipados com CACC por meio de uma rede sem fio. Informações adicionais obtidas por meio dessa rede de comunicação facilitam o projeto de controladores sofisticados que permitem distâncias mais curtas entre os veículos e, ao mesmo tempo, garantem a estabilidade do pelotão [Santini et al. 2015].

O projeto do controlador voltado aos pelotões veiculares depende da topologia de comunicação que determina o tipo de conexão lógica entre os veículos. São exemplos de topologias de comunicação: Predecessor Following (PF), Predecessor-Leader Following (PLF), Bidirectional (BD) e Bidirectional-Leader (BDL) [Zheng et al. 2016]. A topologia mais comumente usada é a PF, na qual o veículo seguidor considera apenas informações de seu antecessor, recebidas através de sensores ou através da rede veicular. 
Na topologia do PLF, o líder se comunica diretamente com todos os veículos seguidores e todos os outros veículos também consideram as informações de seu antecessor para calcular suas ações de controle. Nesta topologia, o controlador pode levar em consideração dois sinais de erro à medida que cada veículo se comunica com seu antecessor e com o líder do pelotão. Na comunicação bidirecional (BD), cada veículo se comunica apenas com seus veículos predecessores e sucessores; enquanto na topologia do BDL, além da comunicação bidirecional, cada veículo se comunica diretamente com o líder do pelotão.

\section{Trabalhos Relacionados}

[Rajamani et al. 2000] propuseram um controlador de pelotões para o projeto PATH. O CACC-PATH é considerado um marco nessa área de pesquisa [Santini et al. 2017] e obteve resultados satisfatórios em simulações comparativas com abordagens mais recentes. O controlador utiliza a topologia PLF, porém, em estudos mais recentes foi utilizado com outras topologias como, BD e PF [Santini et al. 2017]. A solução proposta não trata atrasos na comunicação e não foi projetado para prover confiabilidade na comunicação.

[Wang et al. 2014] buscaram resolver o problema de consenso em pelotões veiculares, por meio de um controle longitudinal. O CACC proposto utiliza uma estratégia de controle de consenso não ponderada, mas restrita, sob a topologia de rede de comutação Markoviana. O trabalho analisou a estabilidade do pelotão formado, porém, não se preocupa em desenvolver uma solução de comunicação confiável para o sistema. Além disso, a topologia de comunicação é definida a priori durante todos experimentos.

Em [Öncü et al. 2014], os autores abordam o projeto que incorpora o efeito de atrasos na amostragem, que ocorrem devido à comunicação sem fio. Os experimentos avaliaram as propriedades de estabilidade do pelotão para variações de headways e atrasos na comunicação. Nos experimentos, foram utilizados apenas dois veículos, a topologia PLF e a troca de informação teve frequência de $25 \mathrm{~Hz}$.

Os autores em [di Bernardo et al. 2016] propuseram um controle longitudinal de pelotões baseado em um problema de consenso de alta ordem para uma rede de agentes dinâmicos na presença de atrasos heterogêneos. A solução tem sua topologia definida $a$ priori como PLF. Os experimentos foram realizados em um pelotão com três veículos. Neste cenário restrito, a solução foi eficaz ao manter o pelotão estável e foi capaz de atenuar a propagação de perturbações. No trabalho, a confiabilidade da comunicação entre os veículos não é considerada.

O trabalho [Zhang and Orosz 2016] investigou os efeitos de veículos com e sem CACC, pertencentes ou não ao pelotão, e atrasos de informação sobre a dinâmica de um pelotão veicular. A topologia utilizada foi a PLF e alguns aspectos da simulação foram simplificados, como os parâmetros de comportamento dos condutores, que foram uniformes e constantes. Por fim, o estudo comprovou ser possível prover estabilidade a um pelotão em certos cenários, entretanto, o controlador não foi capaz de evitar colisões entre os veículos pertencentes ao pelotão.

Em [Zheng et al. 2016], foi avaliada a influência das topologias BD e BDL com atraso fixo na estabilidade e escalabilidade de pelotões veiculares homogêneos. Um modelo linearizado da dinâmica longitudinal do veículo foi derivado usando a técnica de linearização exata por realimentação de estados, que acomoda o atraso inercial da dinâmica do veículo. Apenas simulações numéricas foram usadas na avaliação. 
Em [Schwartz et al. 2012] foi proposto o protocolo de comunicação DOT. A abordagem foca no problema da escalabilidade em redes densas na presença de beacons periódicos. Já em [Chitra and Sathya 2016], os autores integraram a abordagem de controle presente em [Rajamani et al. 2000], com a solução de comunicação DOT. Os autores implementaram um pelotão com quatro veículos e topologia $\mathrm{BD}$, contudo eles não investigaram o impacto que transmissões oriundas de veículos não pertencentes ao pelotão podem provocar em um cenário com alto fluxo de veículos.

O TESTCC [Santini et al. 2017] considera o pelotão como um problema de consenso de segunda ordem, que representa uma topologia de comunicação não fixa com atrasos variáveis no tempo. Os resultados obtidos mostraram que, o uso de um mecanismo de entrega confiável melhora a segurança geral do sistema, quando comparado a abordagens não adaptativas. A solução foi comparada com o ACC padrão e o CACC PATH [Rajamani et al. 2000] em um pelotão de oito veículos.

Em [Lyamin et al. 2018], foi investigado o impacto da implementação da Norma EN 302 637-2 na estabilidade de pelotões veiculares. O controlador usado é baseado em outras abordagens da literatura com algumas simplificações no modelo de controle, como a velocidade do pelotão ser constante, não haver distância mínima entre veículos e a reação dos condutores ser instantânea. Os autores concluíram que a norma estudada gera sobrecarga na rede por conta da disseminação de mensagens de controle, desta forma, eles defendem a implementação de controle de carga em cenários voltados aos pelotões.

No trabalho [Nunen et al. 2019], os autores propuseram uma solução de controle preditivo em combinação com um projeto de controle de feedforward. Essa solução é aplicada em um pelotão heterogêneo de veículos com topologia PLF. Os autores definem que as condições necessárias para que haja estabilidade no pelotão estudado sigam as condições operacionais nominais (sem frenagem de emergência, levando a situações inseguras e saturações de atuadores). No trabalho não são levadas em consideração métricas ligadas à confiabilidade na comunicação.

Na Tabela 1 é apresentada a comparação das abordagens descritas, considerando as seguintes características: (i) co-projeto (ganho do controlador de acordo com informações provenientes do protocolo de comunicação); (ii) topologia de comunicação escolhida; (iii) se o projeto do controlador leva em consideração atrasos na comunicação; (iv) quais fluxos veiculares foram usados nas experimentos realizados; e (vi) se a solução implementa algum mecanismo de controle de carga de mensagens de controle.

Todos os trabalhos analisados usam algum mecanismo de seleção de candidatos para a retransmissão de informação. Somente dois trabalhos investigaram seu desempenho em mais de uma topologia de comunicação e seis consideram o atraso na comunicação. As soluções pesquisadas não levam em consideração informações do protocolo de comunicação para o cálculo dos coeficientes de amortecimento e rigidez do controlador. Nenhum dos trabalhos analisou as abordagens de controle em cenários com fluxo veicular denso, e também nenhum deles implementa algum tipo de controle de carga para diminuir a quantidade de mensagens.

$\mathrm{Na}$ Tabela 1, as soluções mais relevantes, selecionadas por suas características ou pelo alto número de citações, são destacadas em negrito. Essas soluções foram simuladas e seus resultados comparados aos resultados obtidos com o AddP-CACC, na Seção 5. 
Tabela 1. Comparação entre trabalhos relacionados

\begin{tabular}{lccccc}
\hline $\begin{array}{l}\text { Trabalhos } \begin{array}{l}\text { Selecionados } \\
\text { (ordem cronológica) }\end{array} \\
\text { [Rajamani et al. 2000] }\end{array}$ & $\begin{array}{c}\text { Co-projeto } \\
\text { Parametros } \\
\text { Variantes }\end{array}$ & $\begin{array}{c}\text { Topologia } \\
\text { de } \\
\text { Com. }\end{array}$ & $\begin{array}{c}\text { Atraso } \\
\text { na } \\
\text { Com. }\end{array}$ & $\begin{array}{c}\text { Fluxo } \\
\text { Veic. } \\
\text { Util. }\end{array}$ & $\begin{array}{c}\text { Controle } \\
\text { de } \\
\text { Carga }\end{array}$ \\
\hline [Wang et al. 2014] & Não & PLF & Não & N & Não \\
\hline [Öncï et al. 2014] & Não & PLF & Sim & ME & Não \\
\hline [di Bernardo et al. 2016] & Não & PLF & Não & ME & Não \\
\hline [Zhang and Orosz 2016] & Não & PLF & Sim & N & Não \\
\hline [Zheng et al. 2016] & Não & BD e BDL & Não & ME & Não \\
\hline [Chitra and Sathya 2016] & Não & BD & Sim & E & Não \\
\hline [Santini et al. 2017] & Não & PF, PLF e BD & Sim & N & Não \\
\hline [Lyamin et al. 2018] & Não & PLF & Não & ME & Não \\
\hline [Nunen et al. 2019] & Não & PLF & Sim & ME & Não \\
\hline AddP-CACC & Sim & PF, PLF e BD & Sim & ME-D & Sim \\
\hline
\end{tabular}

Muito Esparso (ME): 500, 600 e 800 v/h, Esparsos (E): 1000 e 2000 v/h, Normal (N): 3000 e 4000 v/h e Denso (D): 5000 v/h.

\section{Solução Proposta: AddP-CACC (Adaptive Data Dissemination Protocol)}

Os elementos fundamentais de um sistema CACC são os nós da rede (veículos) e, neste artigo, assume-se que cada veículo possui uma identidade única baseada no endereço MAC do seu módulo de rede. Os veículos participantes possuem componentes que permitem a comunicação e execução de aplicações como; sensores, unidades de armazenamento, unidade de comunicação sem fio e sistema de posicionamento (GPS). Também é assumido que os eventos sempre podem ser detectados pelos sensores presentes nos veículos e que o GPS fornece precisão suficiente para se definir onde e quando ocorreram.

O objetivo geral deste artigo é descrever uma abordagem de co-projeto que fornece confiabilidade à comunicação e estabilidade para formações de pelotões de veículos. A solução proposta consiste de um protocolo de comunicação confiável integrado a um controle adaptativo e cooperativo baseado em consenso, onde o controlador lida com informações provenientes da rede de comunicação e sensoriamento. A estratégia proposta de controle de pelotões de veículos é baseada em princípios de cooperação que servem como parâmetros para garantir o consenso no grupo de veículos. Esta seção apresenta o protocolo de comunicação confiável, o modelo matemático utilizado para representar a dinâmica longitudinal de cada veículo pertencente ao pelotão, a metodologia usada para descrever a solução de controle e, por fim, o modelo de malha fechada que representa a dinâmica do erro de espaçamento entre os veículos de pelotão.

\subsection{Protocolo de Comunicação: AddP}

Para controlar um pelotão veicular, os veículos participantes devem trocar informações ao longo do caminho. Portanto, é necessário um protocolo de comunicação robusto e eficiente, focado na transmissão de dados para ambientes veiculares. Idealmente, esse protocolo deve ser totalmente integrado ao projeto de controle para que ele possa receber dinamicamente feedback sobre o comportamento da rede. O protocolo também deve atenuar questões como nó oculto, broadcast storm, alta latência e oferecer adaptabilidade a vários cenários com densidade veicular variável.

Com este objetivo, a solução proposta adaptou o protocolo de disseminação de dados confiável chamado AddP (Adaptive Data Dissemination Protocol) 
[Oliveira et al. 2017]. O AddP utiliza a densidade, distância e direção dos nós vizinhos para selecionar os veículos que retransmitirão as mensagens de alerta. O AddP baseia-se em uma abordagem de beacons periódicos, as chamadas mensagens de consciência cooperativa (CAMs). A troca de beacons permite que os nós (veículos) notifiquem outros nós, que estão localizados a um salto de distância, de sua presença e seu status. Estes beacons contêm a identificação do veículo, a densidade local, a velocidade, a posição e a outras informações pertinentes a seus vizinhos. No entanto, quando ocorre congestionamento no canal (por exemplo, numa rede densa), é necessário reduzir a carga de beacons para manter uma certa quantidade de largura de banda disponível para as mensagens de aviso, porque transportam informações críticas de grande importância. Portanto, o AddP usa o mecanismo chamado de Adaptative Beacon Congestion Control para evitar sobrecarga na comunicação do pelotão e para garantir que a transmissão de um alerta no momento da frenagem seja realizada sem interferências.

Todos os veículos usam ainda o mecanismo Disseminated Messages Monitoring para verificar se um nó selecionado retransmitiu o aviso atual. Caso contrário, o vizinho transmite a mensagem de aviso. Esse mecanismo é usado para resolver o problema do nó oculto e aumentar a confiabilidade da disseminação em cenários de densidade esparsa.

\subsection{Controle Cooperativo e Adaptativo}

A ideia do controlador proposto é considerar o pelotão como um problema de consenso de alta ordem, responsável por uma topologia de comunicação não fixa com atrasos no tempo. O consenso distribuído é uma maneira de abordar os NCSs. Em um problema de consenso, todos os nós em uma rede devem chegar a um acordo. Ou seja, no estado estacionário todos devem convergir para o mesmo objetivo. No caso particular de um pelotão de veículos, busca-se ter a mesma distância entre pares de veículos consecutivos.

Ao considerar um pelotão com $n$ veículos seguidores e um veículo líder, podese representar o fluxo de informação entre os seguidores por uma topologia de grafo direcionado $\mathcal{G}=(\mathcal{V}, \mathcal{E})$, tal que $\mathcal{V}=\left\{\alpha_{1}, \alpha_{2}, \ldots, \alpha_{n}\right\}$ é o conjunto de $n$ vértices e $\mathcal{E}$ representa o conjunto de arestas que os conectam, dadas por $a_{i j}=\left(\alpha_{i}, \alpha_{j}\right)$. O vértice $\alpha_{i}$ representa o $i$-ésimo veículo do pelotão e cada aresta $a_{i j}$ representa a existência de um canal de comunicação direcionado do veículo $i$ para o veículo $j$. Para representar o fluxo de informações entre os seguidores e entre os seguidores e o líder, considera-se um grafo aumentado $\tilde{\mathcal{G}}=(\tilde{\mathcal{V}}, \tilde{\mathcal{E}})$, tal que $\tilde{\mathcal{V}}=\left\{\alpha_{0}, \alpha_{1}, \ldots, \alpha_{n}\right\}$, no qual $\alpha_{0}$ é o veículo líder.

\subsubsection{Dinâmica Longitudinal do Veículo}

Assumindo-se um modelo simplificado por meio da segunda lei de Newton, ao se mover no plano, um veículo sofre ação de forças externas, tais como: de arrasto, gravitacional, longitudinal dos pneus e de atrito dos pneus. Neste trabalho, foi usado o modelo definido por [Rajamani 2011] para retratar o torque no veículo proveniente dos seus atuadores. Também assume-se que cada veículo é dotado de uma lei de controle linearizante. Ao realizar as substituições e simplificações, o modelo linearizado para a dinâmica longitudinal do veículo é expresso como: $u(t)=\varsigma \dot{a}(t)+a(t)$, onde $a(t)$ é a aceleração do veículo; $\varsigma$ denota o atraso inercial do powertrain; e $u(t)$ é a entrada de controle. Ao escolher a posição de cada veículo $p_{i}(t)$, velocidade $v_{i}(t)$ e aceleração $a_{i}(t)$ como o estado, um modelo de terceira ordem é derivado para cada veículo: $\dot{x}_{i}(t)=A x_{i}(t)+B u_{i}(t)$. 


\subsubsection{Política de Espaçamento}

Ao considerar os parâmetros relativos a qualquer par de veículos no pelotão, a distância entre os dois veículos adjacentes (parados) pode ser expressa tanto em termos dos comprimentos dos veículos, quanto da distância de segurança requerida. A distância entre os veículos $i$ e $j$ pode ser reformulada em relação ao veículo principal como $\hat{d p}_{i j}=$ $\hat{d p}_{i 0}-\hat{d p}_{j 0}$. Além disso, destaca-se que $d p_{i j}=-d p_{j i}$. Neste trabalho, assume-se que a distância desejada a se seguir é linearmente proporcional à velocidade do líder.

$$
d_{i j}=h_{i j} v_{0}+d p_{i j}
$$

e que o tempo de headway constante do veículo $i$ com relação ao veículo $j$ pode ser calculado a partir do tempo de headway em relação ao veículo líder $h_{i j}=h_{i 0}-h_{j 0}$ (por exemplo, com veículos em movimento na mesma velocidade constante).

\subsubsection{Modelagem do Pelotão como Problema de Consenso}

Ao considerar um pelotão veicular, o comportamento do $i$-ésimo veículo é matematicamente descrito como o seguinte agente inercial $(i=1, \ldots, n)$ :

$$
\dot{p}_{i}(t)=v_{i}(t), \quad \dot{v}_{i}(t)=a_{i}(t) \quad e \quad \dot{a}(t)=\frac{-a_{i}(t)+u_{i}(t)}{\varsigma},
$$

onde $p_{i}(t)(\mathrm{m}), a_{i}(t)\left(\mathrm{m} / \mathrm{s}^{2}\right) v_{i}(t)(\mathrm{m} / \mathrm{s})$ e $u_{i}(t)$ indicam que a entrada de controle deve ser escolhida apropriadamente para alcançar a posição desejada, a manutenção da velocidade e as manobras de frenagem.

O modelo linear de veículo da Equação 2 pode ser derivado ao aplicar a linearização input-output (I/O) para simplificar a complexidade do modelo que descreve a dinâmica longitudinal de veículo. Por fim, o vetor de estado do $i$-ésimo veículo é definido como $x_{i}(t)=\left[x_{i}^{(1)}(t), x_{i}^{(2)}(t), x_{i}^{(3)}(t)\right]^{\top}=\left[p_{i}(t), v_{i}(t), a_{i}(t)\right]^{\top} \in \mathbb{R}^{2}$. Ao supor que o pelotão tenha que se mover com uma velocidade constante de referência, $v_{0}$, a dinâmica de referência do líder pode ser descrita como:

$$
\dot{p}_{0}(t)=v_{0}(t), \quad \dot{v}_{0}(t)=a_{0}(t) e \quad \dot{a}_{0}(t)=0 .
$$

Pode-se definir como $x_{0}(t)=\left[p_{0}(t), v_{0}, a_{0}\right]^{\top} \in \mathbb{R}^{2}$ o vetor de estado líder. Dadas as Equações 2 e 3, o problema de manter uma política de espaçamento interveicular desejada e uma velocidade comum do pelotão de veículos pode ser reescrito como um problema de consenso de terceira ordem. O objetivo do controle do pelotão é manter a mesma velocidade do líder e manter um espaço desejado entre veículos:

$$
\lim _{t \rightarrow+\infty}\left|v_{i}-v_{0}\right|=0 \quad e \quad \lim _{t \rightarrow+\infty}\left|p_{i}-p_{i-1}-d_{i i-1}\right|=0
$$

onde $d_{i i-1}$ representa a distância desejada entre o veículo $i$ e o veículo $i-1$. A distância desejada $d_{i i-1}$ pode ser expressa ao utilizar o tempo de headway $h_{i i-1}$ e a distância de paragem $d p_{i i-1}$, como mostrada na Equação 1. A síntese do controlador local para o nó $i$ só pode usar as informações dos nós no conjunto $\mathcal{I}_{i}=\mathcal{C}_{i} \cup \mathcal{P}_{i}$. Para definir a lei de controle, pode-se assumir que a comunicação é ideal, desta forma, tem-se o seguinte controle local para o veiculo $i$ :

$$
u_{i}=-\sum_{j \in \mathcal{I}}\left[k_{p}\left(p_{i}-p_{j}-d_{i, j}\right)+k_{v}\left(v_{i}-v_{j}\right)+k_{a}\left(a_{i}-a_{j}\right)\right],
$$


no qual $k_{p}, k_{v}$ e $k_{a}$ são coeficientes de rigidez e amortecimento a serem oportunamente ajustados para regular o comportamento entre os veículos vizinhos. Por fim, pode-se representar a forma compacta da Equação 5, por:

$$
u_{i}=-k^{\top} \sum_{j \in \mathcal{I}}\left(x_{i}-x_{j}\right), \quad i=1, \ldots, n,
$$

onde $k=\left[k_{p}, k_{v}, k_{a}\right]^{\top}$ é um vetor contendo os ganhos do controlador, $x_{i}(t)=\left[p_{i}, v_{i}, a_{i}\right]^{\top}$ é o vetor dos estados de cada veículo e $u_{i}(t)$ a lei de controle. Para tentar obter uma sintonia de controlador que apresente o desempenho desejado no cenário de referência, recorreu-se à sintonia por tentativas e erros. Os ganhos foram definidos de acordo com os intervalos de atrasos possíveis no cenário $(0 \mu s$ a $10 \mu s)$. Os valores para $k_{p}, k_{v}$ e $k_{a}$ foram definidos de acordo com:

$$
k_{p}=\left\{\begin{array}{ll}
0.9, & \text { if } 0 \mu s \leq \tau \leq 2 \mu s \\
1, & \text { if } \tau>2 \mu s
\end{array}, \quad k_{v}=\left\{\begin{array}{ll}
1, & \text { if } 0 \mu s \leq \tau \leq 2 \mu s \\
0.7, & \text { if } \tau>2 \mu s
\end{array} \text { and } \quad k_{a}= \begin{cases}1, & \text { if } \tau \geq 0 .\end{cases}\right.\right.
$$

\subsection{Dinâmica do Pelotão}

Para analisar a dinâmica de formação do pelotão, é definido um vetor de estados composto pelos erros dos estados dos veículos em relação ao líder:

$$
\bar{x}_{i}(t)=\left[\begin{array}{c}
\bar{p}_{i}(t) \\
\bar{v}_{i}(t) \\
\bar{a}_{i}(t)
\end{array}\right]=\left[\begin{array}{c}
p_{i}(t)-p_{i}^{*}(t) \\
v_{i}(t)-v_{0}(t) \\
a_{i}(t)-a_{0}(t)
\end{array}\right],
$$

onde $p_{i}^{*}(t)=p_{0}(t)-d_{0 i}=p_{0}(t)-h_{0 i} v_{0}-d p_{0 i}$ é a posição desejada do veículo $i$. Diante disso, a lei de controle de cada veículo pode ser definida como:

$$
u_{i}=-\sum_{j \in \mathcal{I}_{i}}\left[k_{p}\left(p_{i}(t)-p_{i}^{*}(t)-p_{j}(t)-p_{j}^{*}(t)\right)+k_{v}\left(v_{i}(t)-v_{0}(t)-v_{j}(t)-v_{0}(t)\right)+k_{a}\left(a_{i}(t)-a_{0}(t)-a_{j}(t)-a_{0}(t)\right)\right] \text { (9) }
$$

Ao utilizar o vetor de estados composto pelos erros dos estados dos veículos em relação ao líder (Equação 8) e realizar as simplificações possíveis, tem-se:

$$
u_{i}=-k^{\top} \sum_{j=1}^{n}\left[a_{i j}\left(\bar{x}_{i}(t)-\bar{x}_{j}(t)\right)+\wp_{i}\left(\bar{x}_{i}(t)-\bar{x}_{0}(t)\right)\right] .
$$

Desta forma, a dinâmica de erro em malha-fechada do $i$-ésimo veículo é expressa por $\dot{\bar{x}}_{i}(t)=A \bar{x}_{i}(t)+B u_{i}(t)$. Ao substituir $u_{i}(t)$ na dinâmica de erro em malha-fechada, pela Equação 10, tem-se:

$$
\dot{\bar{x}}_{i}(t)=A \bar{x}_{i}(t)+B k^{\top} \sum_{j=1}^{n}\left[a_{i j}\left(\bar{x}_{i}(t)-\bar{x}_{j}(t)\right)+\wp_{i}\left(\bar{x}_{i}(t)-\bar{x}_{0}(t)\right)\right] .
$$

Para avaliar a influência do atraso de comunicação na dinâmica do pelotão é apresentado o modelo matemático para a dinâmica do erro na formação de um pelotão sujeito a atraso na comunicação. Ao analisar a lei de controle representada pela Equação 5, notase que o veículo $i$ necessita dos valores em tempo real da posição, velocidade e aceleração dos veículos adjacentes que se comunicam com $i$. Entretanto, no caso da existência de atraso de comunicação entre os veículos, a lei de controle (Equação 5), passa a ser representada pela Equação 12:

$$
u_{i}=-\sum_{j \in \mathcal{I}}\left[k_{p}\left(p_{i}(t)-p_{j}\left(t-\tau_{i j}\right)-d_{i j}\right)+k_{v}\left(v_{i}(t)-v_{j}\left(t-\tau_{i j}\right)\right)+k_{a}\left(a_{i}(t)-a_{j}\left(t-\tau_{i j}\right)\right)\right],
$$

onde $\tau$ representa o atraso variável na comunicação, que afeta o $i$-ésimo veículo quando a informação é transmitida de seu vizinho $j$ e do líder do pelotão, respectivamente.

$\mathrm{O}$ atraso $\tau_{i j}(t)$ pode ser considerado entre um valor máximo e mínimo, por exemplo $0 \leq \tau_{i j}(t) \leq \tau_{\max }\left(\tau_{i j}(t)\right.$ é uma função contínua por partes). Pode-se observar que 
algumas das informações transmitidas, como o estado do veículo anterior, podem estar disponíveis a partir de medições realizadas por sensores a bordo. A entrega dessas informações não será afetada por atrasos sensíveis, e pode-se assumir $\tau_{i j}(t)=0$. Além disso, embora o atraso $\tau_{i j}(t)$ seja desconhecido, pode ser detectado e avaliado de forma local em todos os veículos do pelotão, já que os nós recebem beacons com o timestamp relativo $\bar{t}$, que representa o instante em que a informação é medida.

Como visto anteriormente, a dinâmica de erro em malha-fechada do $i$-ésimo veículo é definida por $\dot{\bar{x}}_{i}(t)=A \bar{x}_{i}(t)+B u_{i}(t)$. Realizando substituições e simplificações:

$$
\dot{\overline{x_{i}}}=A \bar{x}_{i}-B k^{\top} \sum_{j=1}^{n}\left[a_{i j}\left(\bar{x}_{i}(t)-\bar{x}_{j}(t)\right)+\wp_{i}\left(\bar{x}_{i}(t)-\bar{x}_{0}(t)\right)+a_{i j}\left(x_{j}(t)-x_{j}(t-\tau)\right)+\wp_{i}\left(x_{i}(t)-x_{i}(t-\tau)\right)\right]
$$

Por fim, a dinâmica do erro sob atraso em malha-fechada, de acordo com os apontamentos feitos na Seção 4.2, pode ser expressa pela Equação 14.

$$
\dot{\bar{X}}(t)=\left[I_{N} \otimes A-(\mathcal{L}+\mathcal{P}) \otimes B k^{\top}\right] \bar{X}(t)-\left[(\mathcal{A}+\mathcal{P}) \otimes B k^{\top}\right] \int_{-\tau}^{0} \dot{X}(t+s) d s .
$$

\section{Simulação e Análise dos Resultados}

Esta seção descreve o projeto de experimentos realizados para avaliar a solução proposta e apresenta os resultados das simulações com suas respectivas análises. O objetivo dos experimentos foi comparar a AddP-CACC com as soluções selecionadas na Seção 3. Nesse sentido, uma aplicação EEBL (Emergency Electronic Brake Light) foi implementada. A avaliação é dividida em duas partes. Inicialmente, a solução proposta AddP-CACC é comparada a outras soluções no que diz respeito a comunicação confiável para VANETs. Em seguida, a AddP-CACC é avaliada a fim de verificar seu desempenho diante do controle de um pelotão veicular.

\subsection{Parâmetros de simulação e mobilidade}

Um segmento da rodovia BR-101 no sul do Brasil foi modelado, consistindo em uma via com duas pistas para cada direção e comprimento de $5 \mathrm{~km}$. O tráfego diário normal neste trecho é de 60 mil veículos. Em todos os experimentos, os dados são coletados somente após 500 segundos, que é considerado o tempo necessário para o cenário ser preenchido.

Nas experiências, foi utilizado o simulador de rede baseado em eventos OMNeT++ acoplado bidirecionalmente com o simulador de tráfego SUMO (Simulation of Urban Mobility). Foram definidos três tipos de veículos. O pelotão veicular foi constituído por dez carros e com 1 segundo de headway. A distância de paragem foi definida para 10 metros e a constante inercial $\varsigma=0,5$. Os estilos de direção foram escolhidos aleatoriamente dentro de limites aceitáveis. O parâmetro de imperfeição do condutor foi definido usando valores diferentes para a velocidade desejada [Oliveira et al. 2017]. Nas experiências, optou-se por variar as velocidades máximas do veículo (50, 70, 90 e 110 $\mathrm{km} / \mathrm{h}$ ). Na Tabela 2 são apresentados os parâmetros de simulação usados para definir os módulos do framework Veins nos experimentos.

Em todos os cenários simulados de rodovias, havia duas RSUs responsáveis pela propagação de alertas para veículos que não os receberam pela via convencional. Uma delas foi posicionada no início do primeiro quilômetro e a outra no início do terceiro quilômetro. Foram definidos cenários com diferentes fluxos de veículos, simulando, por exemplo, tráfego normal (3000 veículos/hora) e tráfego denso (5000 veículos/hora) ${ }^{1}$.

\footnotetext{
${ }^{1} \mathrm{O}$ tráfego diário e os volumes de fluxos de tráfego foram obtidos na Policia Rodoviária Federal em 2017.
} 
Tabela 2. Parâmetros de simulação (Veins)

\begin{tabular}{ll|ll}
\hline Parâmetros & Valores & Parâmetros & Valores \\
\hline MAC Layer & IEEE $802.11 \mathrm{p} / 1609.4$ & Tamanho do Beacon & 32 bytes \\
\hline Frequência & $5.89 \mathrm{GHz}$ & Atraso Máximo $\tau_{\max }$ & $10 \mu \mathrm{s}$ \\
\hline Raio de transmissão & 256 metros & Modelo de propagação & Two-ray interference \\
\hline Força do sinal / Taxa de bits & $2 \mathrm{~mW} / 18 \mathrm{Mpbs}$ & Modelo de desvanecimento & Nakagami-m \\
\hline Quantidade de repetições & 33 vezes & Tempo de simulação & 15 min \\
\hline
\end{tabular}

\subsection{Avaliação do Desempenho do Procolo de Comunicação}

Devido à limitação de espaço, neste artigo apenas duas métricas de desempenho são apresentadas: total de beacons (TB), que é o total de mensagens de controle enviadas; e taxa de sucesso $(T S)$, que é a porcentagem de veículos que receberam as mensagens enviadas com sucesso.

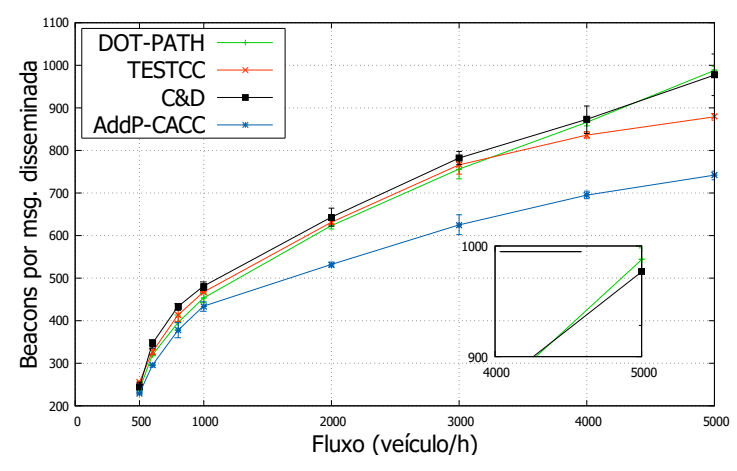

Figura 1. Beacons enviados (TB)

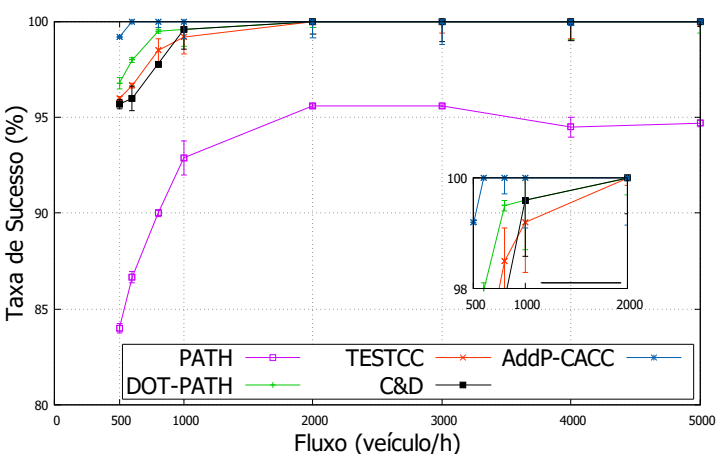

Figura 2. Taxa de sucesso (TS)

Na Figura 1 é ilustrado o total de beacons transmitidos por todos os veículos assumindo diferentes fluxos de tráfego. Diferentemente de outras abordagens, por não possuir mecanismo de controle de carga, a solução PATH atingiu valores superiores a 2000 beacons por mensagem disseminada, desta forma, seus resultados não são apresentados. À medida que o fluxo de veículos aumenta, o AddP-CACC impõe uma menor quantidade de mensagens de controle transmitidas por veículo pois ajusta o tempo entre as retransmissões de beacons com base na densidade do nó para evitar o problema de broadcast storm.

Na Figura 2, é apresentada a taxa de sucesso para todas as abordagens sob diferentes fluxos de tráfego. Quando comparado com as abordagens DOT-PATH, TESTCC, Connect \& Drive e PATH, AddP-CACC é a solução com melhor desempenho mesmo em cenários esparsos. Tais resultados podem ser explicados pelo fato de que o protocolo de comunicação do AddP-CACC usa o mecanismo Disseminated Messages Monitoring para transmitir as mensagens de aviso não entregues no caso de uma partição de rede ou um problema de nó oculto. Em cenários densos, o comportamento de AddP-CACC, DOT-PATH, Connect \& Drive e TESTCC são semelhantes (cobertura de $100 \%$ ).

Com base nos resultados obtidos, pode-se concluir que o AddP-CACC é uma solução adequada para a aplicações de pelotões, as quais requerem entrega confiável de dados em diferentes densidades de rede, e que são sensíveis ao atraso. 


\subsection{Avaliação do Desempenho do Controlador}

Nas experiências realizadas nesta seção, a topologia PLF foi adotada, por permitir que os veículos recebam comunicação tanto do líder como, também, de seus antecessores no cálculo da ação de controle.
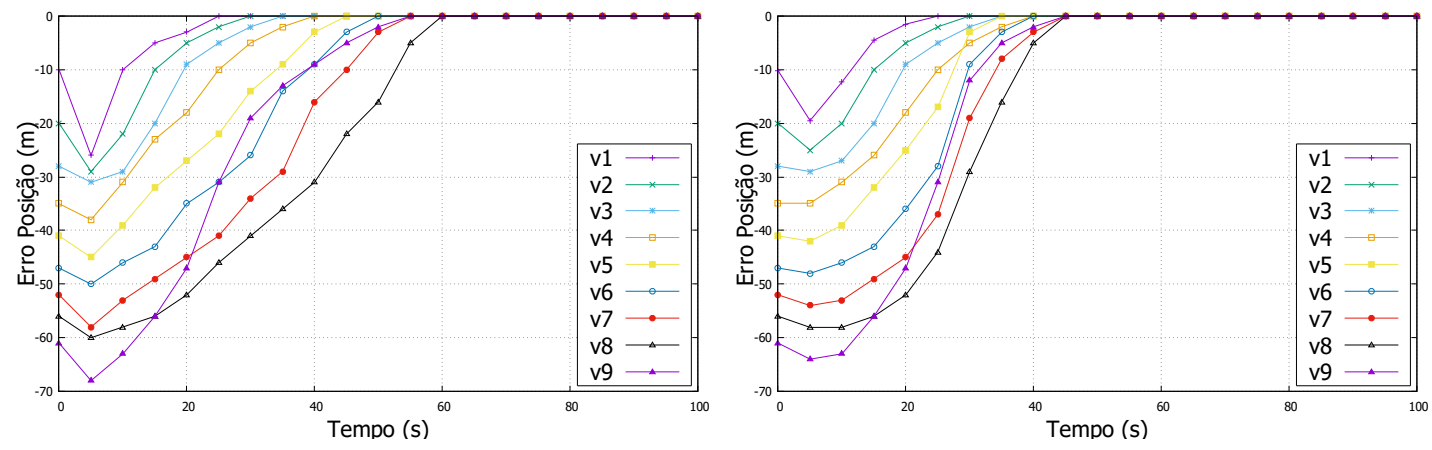

Figura 3. Erros de posicionamento de veículos (v1-9) no pelotão. (a) Fluxo: 3000 veículos/hora; (b) Fluxo: 5000 veículos/hora

\subsubsection{Comportamento em Cenários de Tráfego Normal e de Alta Densidade}

Em um primeiro cenário, o objetivo é observar se o AddP-CACC fornece consenso entre os veículos de pelotão. O líder mantém velocidade constante e os seguidores tentam alcançar o espaçamento desejado entre veículos. Nas Figuras 3 e 4 são apresentados os resultados. É possível confirmar a capacidade do controlador de fornecer consenso e manter o pelotão estável. É importante notar que o AddP-CACC controla a carga, mesmo em uma rede com alta densidade, para que a comunicação no pelotão permaneça estável.
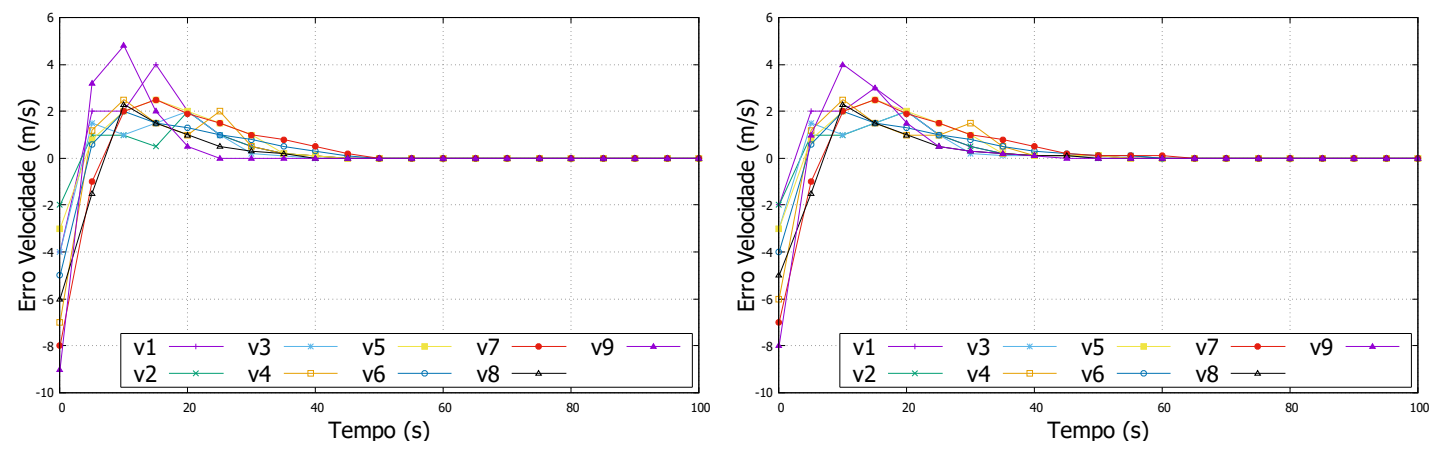

Figura 4. Erros na velocidade do pelotão de veículos (v1-9) comparada com a do lider. (a) Fluxo: 3000 veículos/hora; (b) Fluxo: 5000 veículos/hora

No segundo cenário, verificou-se se os veículos são capazes de acompanhar a velocidade do veículo líder. O líder acelera e desacelera para alterar sua velocidade de cruzeiro e os seguidores devem alterar corretamente sua velocidade. Na Figura 5, o líder acelera para $30,6 \mathrm{~m} / \mathrm{s}$ com uma aceleração constante de $3 \mathrm{~m} / \mathrm{s}^{2}$ e todos os seguidores mudam corretamente seu comportamento, alcançando consenso sobre a velocidade.

Em um terceiro cenário, o veículo líder é forçado a desacelerar de 30,6 m/s para $0 \mathrm{~m} / \mathrm{s}$ (parada completa) para simular uma manobra de frenagem. Os veículos iniciam a simulação com uma distância entre para-choques de 35 metros. Após o veículo líder realizar a frenagem, os veículos seguidores devem manter uma distância de 10 metros. 

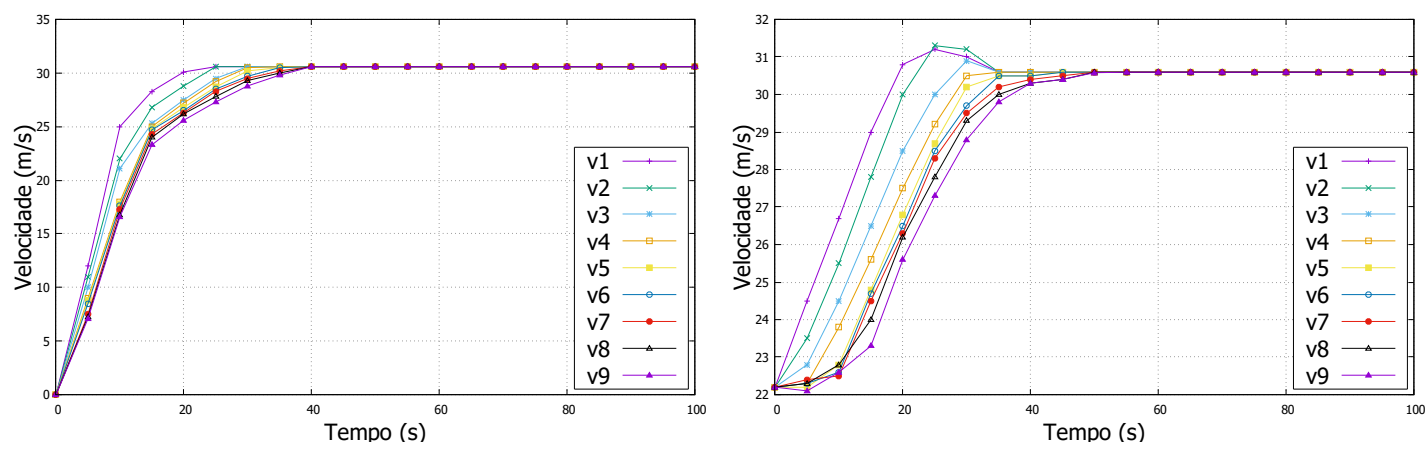

Figura 5. Velocidades ao seguir o líder. (a) 3000 veículos/hora; (b) 5000 veículos/hora

Os resultados apresentados na Figura 6, indicam que todos os veículos param de forma segura e convergem para uma distância de $10 \mathrm{~m}$.
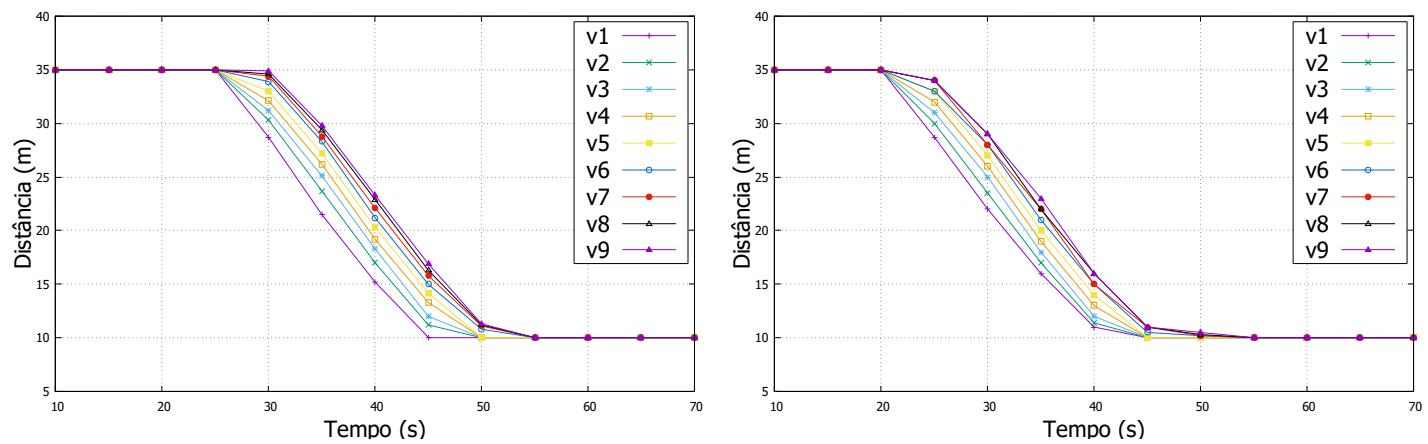

Figura 6. Distância entre para-choques. (a) 3000 veículos/hora; (b) 5000 veículos/hora

No quarto cenário, foi avaliado o consenso da solução proposta, por meio de um distúrbio sinusoidal. O líder acelera e desacelera para formar uma perturbação sinusoidal em torno da velocidade média de $30,6 \mathrm{~m} / \mathrm{s}^{2}$ [Santini et al. 2015]: $\delta(t)=A \cos \left(\frac{6}{100} \pi t\right)$, onde $A=2,7 \mathrm{~m} / \mathrm{s}$. Na Figura 7 são ilustradas as distâncias entre veículos. Observa-se que a perturbação sinusoidal é atenuada a jusante do pelotão veicular, e a oscilação já é quase imperceptível no veículo $v 4$.
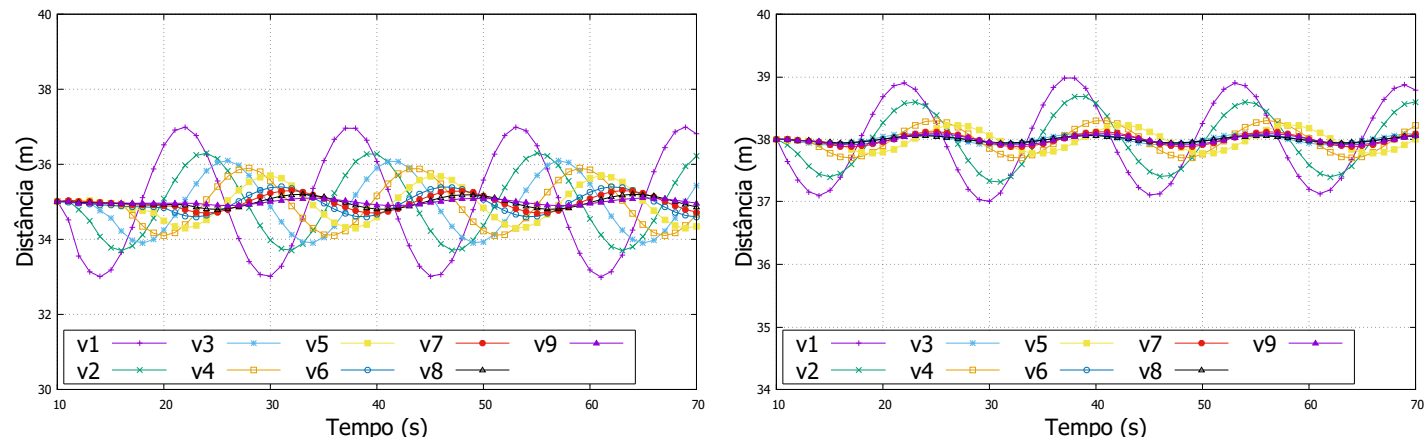

Figura 7. Distúrbios senoidais sobre a velocidade do lider. (a) 3000 veículos/hora; (b) 5000 veículos/hora 


\subsubsection{Comparação entre abordagens de controle}

Nesta seção avaliou-se o desempenho da AddP-CACC diante de outras abordagens, comparando-as em termos de estabilidade em cadeia sob o modelo de propagação TwoRay Interference que captura tanto a atenuação do sinal, quanto os efeitos de reflexão do solo. Também foi levado em consideração o desvanecimento distribuído Nakagami- $m$.

Comparou-se as abordagens por meio de uma medida frequentemente usada na verificação da acurácia de modelos numéricos que é o Erro Quadrático Médio (EQM). O EQM é mais sensível aos grandes erros, por elevar as diferenças individuais ao quadrado, assim busca evitar o cancelamento de erros com sinais diferentes. Por meio dos dados obtidos por simulação, o EQM foi calculado para cada solução (e topologias de comunicação). Um comparativo entre as abordagens é apresentado na Figura 8. A solução proposta AddP-CACC apresenta menor erro quadrático médio relacionado à velocidade nos pontos observados e nas três topologias de comunicação implementadas.
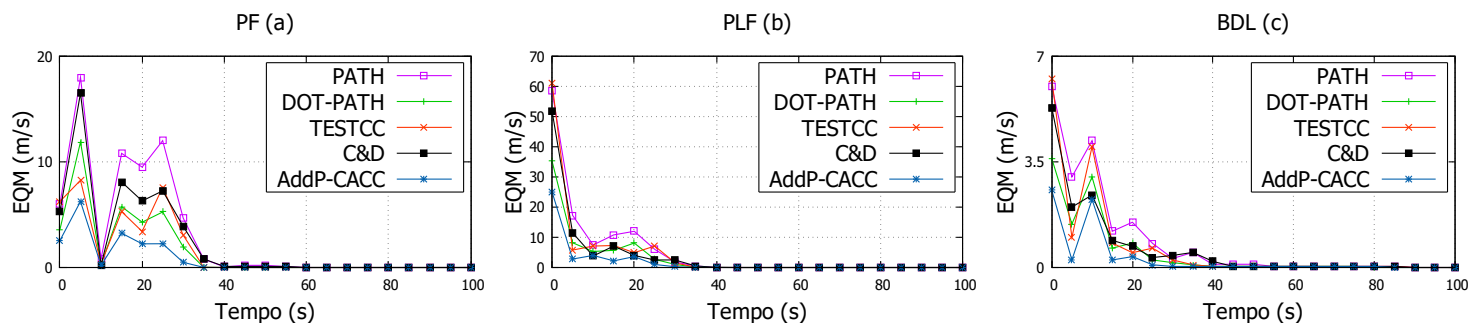

Figura 8. Comparação entre abordagens segundo o EQM. (Fluxo: 3000 veículos/hora)

\section{Conclusões}

Um dos pontos estudados neste artigo foi a comunicação em VANETs e os problemas existentes que podem inviabilizar o controle longitudinal de um pelotão. Foi proposta uma solução que adapta o intervalo entre mensagens de controle de acordo com o fluxo veicular. Também foi proposta uma solução de controle cooperativo decentralizado baseado em consenso na qual, ao contrário das abordagens da literatura, a topologia da comunicação é configurável. Um dos benefícios é a capacidade de adaptar o padrão de comunicação às características da rede. A abordagem foi analisada em cenários desafiadores, incluindo distúrbios de rede, mostrando suas características de robustez, estabilidade e convergência. Como trabalhos futuros, sugere-se estender a solução proposta para que este possa ser adaptado para outros tipos de aplicações de segurança no trânsito e cenários (urbano, por exemplo).

\section{Referências}

Chitra, M. and Sathya, S. S. (2016). Bidirectional data dissemination in vehicular ad hoc networks using epidemic spreading model. In Proc. of the International Conference on Informatics and Analytics, ICIA-16, pages 57:1-57:8, New York, NY, USA. ACM.

di Bernardo, M., Falcone, P., Salvi, A., and Santini, S. (2016). Design, analysis, and experimental validation of a distributed protocol for platooning in the presence of timevarying heterogeneous delays. IEEE Trans. on Contr. Systems Tech., 24(2):413-427.

Elefteriadou, L. (2013). An Introduction to Traffic Flow Theory. Springer Optimization and Its Applications. Springer New York. 
Hicks, D. J. (2018). The safety of autonomous vehicles: Lessons from philosophy of science. IEEE Technology and Society Magazine, 37(1):62-69.

Kheirandish, F., Ekramian, M., and Ataei, M. (2017). Stability analysis of networked control systems with time varying delays. In 2017 Iranian Conference on Electrical Engineering (ICEE), pages 633-638.

Lyamin, N., Vinel, A., Jonsson, M., and Bellalta, B. (2018). Cooperative awareness in VANETs: On ETSI EN 302 637-2 performance. IEEE Trans. on Vehicular Tech., 67(1):17-28.

Nunen, E., Reinders, J., Semsar-Kazerooni, E., and van de Wouw, N. (2019). String stable model predictive cooperative adaptive cruise control for heterogeneous platoons. IEEE Transactions on Intelligent Vehicles, 4(2):186-196.

Oliveira, R., Montez, C., Boukerche, A., and Wangham, M. S. (2017). Reliable data dissemination protocol for vanet traffic safety applications. Ad Hoc Networks, 63:30 44.

Rajamani, R. (2011). Vehicle Dynamics and Control. Mechanical Engineering Series. Springer US.

Rajamani, R., Han-Shue Tan, Boon Kait Law, and Wei-Bin Zhang (2000). Demonstration of integrated longitudinal and lateral control for the operation of automated vehicles in platoons. IEEE Trans. on Cont. Systems Technology, 8(4):695-708.

Santini, S., Salvi, A., Valente, A. S., Pescapè, A., Segata, M., and Cigno, R. L. (2015). A consensus-based approach for platooning with inter-vehicular communications. In 2015 IEEE Conf. on Computer Communications (INFOCOM), pages 1158-1166.

Santini, S., Salvi, A., Valente, A. S., Pescapè, A., Segata, M., and Cigno, R. L. (2017). A consensus-based approach for platooning with intervehicular communications and its validation in realistic scenarios. IEEE Trans. on Vehicular Tech., 66(3):1985-1999.

Schwartz, R. S., Das, K., Scholten, H., and Havinga, P. (2012). Exploiting beacons for scalable broadcast data dissemination in vanets. In Proceedings of the Ninth ACM International Workshop on Vehicular Inter-networking, Systems, and Applications, VANET '12, pages 53-62, New York, NY, USA. ACM.

Wang, L. Y., Syed, A., Yin, G. G., Pandya, A., and Zhang, H. (2014). Control of vehicle platoons for highway safety and efficient utility: Consensus with communications and vehicle dynamics. Journal of Systems Science and Complexity, 27(4):605-631.

Zhang, L. and Orosz, G. (2016). Motif-based design for connected vehicle systems in presence of heterogeneous connectivity structures and time delays. IEEE Transactions on Intelligent Transportation Systems, 17(6):1638-1651.

Zheng, Y., Li, S. E., Wang, J., Cao, D., and Li, K. (2016). Stability and scalability of homogeneous vehicular platoon: Study on the influence of information flow topologies. IEEE Transactions on Intelligent Transportation Systems, 17(1):14-26.

Öncü, S., Ploeg, J., van de Wouw, N., and Nijmeijer, H. (2014). Cooperative adaptive cruise control: Network-aware analysis of string stability. IEEE Transactions on Intelligent Transportation Systems, 15(4):1527-1537. 